\title{
Dermatological Emergencies: Current Trends in Management
}

\author{
Falodun $O$ and Ogunbiyi $A$
}

Dermatology Unit, Department of Medicine, University College Hospital, Ibadan, Nigeria.

\begin{abstract}
SUMMARY
Emergencies in dermatology are well recognized and are associated with significant morbidity and mortality. Early recognition of these conditions with institution of prompt medical care can help in reducing the morbidity and mortality associated with these conditions. This article reviews relevant dermatologic emergencies with respect to this environment with emphasis on current trends in management. Prompt and aggressive management of dermatologic emergencies are important to reduce mortality related to these skin disorders so as to prevent skin failure. Dermatologic emergencies are clinical conditions which lead to increased morbidity and mortality. Recognizing them and the urgency required in their management would help in reducing the attendant skin failure that may arise from these conditions.
\end{abstract}

Keywords: Dermatologic emergency, skin failure, management.

\section{INTRODUCTION}

Dermatologic emergencies are a well recognized group of disorders requiring prompt treatment. They could all lead to acute skin failure with its attendant sequelae such as sepsis, multi organ failure and pulmonary embolism.

Skin failure is an emerging concept which refers to a state of total dysfunction of the skin resulting from different aetiological factors $[1,2]$. There is loss of normal temperature control with inability to maintain the core body temperature. There is also percutaneous fluid, electrolyte and protein

\section{All Correspondence to Dr A. Ogunbiyi}

Dermatology Unit,Department of Medicine,

University College Hospital, Ibadan

E-mail:adebolaogunbiyi@yahoo.com loss resulting in various complications. The barrier mechanism against foreign materials is also compromised [1,2].

Dermatologic emergencies to be discussed include the following:

1. Stevens Johnson syndrome-Toxic epidermal necrolysis

2. Pemphigus vulgaris

3. Staphylococcal scalded skin syndrome

4. Lepra reaction

5. Angioedema

The effective management of each of the above listed conditions is possible only when the underlying pathogenic mechanism is clear to the treating clinician.

\section{TOXIC EPIDERMAL NECROLYSIS AND STEVENS JOHNSON'S SYNDROME}

Both Steven Johnson's syndrome (SJS) and Toxic Epidermal Necrolysis (TEN) are blistering conditions of the skin which may result in high morbidity and mortality. It is believed that they both represent different spectra of the same disease. However, some feel they are different disease entities. TEN was first described by Alan Lyell in 1956 [3, 4]. TEN is the most severe form of cutaneous drug reaction. It is a rapidly evolving mucocutaneous reaction characterized by widespread erythema, necrosis and bullous detachment of extensive areas of the epidermis. Steven Johnson's would be described as erythema multiforme with more than one mucous membrane involvement. TEN represents the most severe variant of a disease spectrum that includes bullous erythema multiforme and Stevens Johnson syndrome.

The differentiation between toxic epidermal necrolysis and Stevens Johnson syndrome is mainly clinical. In TEN, the disease is more severe with epidermal detachment greater than $30 \%[5,6]$ while in Stevens Johnson syndrome, epidermal 
detachment is less that $10 \%$ of body surface area. The patient in Stevens-Johnson's syndrome also has target lesions $[5,6]$.

\section{Aetio-Pathogenesis}

The causes of the two conditions are numerous. Drugs are the major precipitating cause of TEN and SJS. Viral infections have also been implicated in the aetiology of these two conditions, especially in patients who have recurrent SJS in the absence of drug use. Common drugs implicated include sulphonamides, quinolones, anticonvulsants like phenobarbitone, phenytion and carbamazepine. Listed as a possible cause but very rarely seen in our environment is erythromycin [21, 22].

Most likely mechanism favoured is an immune complex reaction. One theory suggests that accumulation of drug metabolites in the epidermis in genetically predisposed individuals induces an immunologic process analogous to that which occurs in graft versus host disease. $\mathrm{CD}_{8}{ }^{+} \mathrm{T}$ lymphocytes and macrophages activate an inflammatory cascade, leading to widespread apoptosis of epidermal cells.

\section{Clinical Features}

Patients with TEN and SJS are usually ill and may present with pyrexia $[6,7,8,9]$. Examination of the skin may show generalized papular exanthem, purpuric macules, atypical target lesions, bullae or erosions.

Skin lesions rapidly coalesce in patients with TEN producing large blisters. The lesions separate with light pressure (Nikolsky sign) in patients with TEN and the underlying denuded skin is erythematous [5, 6, 7]. Involvement of mucosa, oral, ocular, genital and respiratory systems vary in the two conditions and this leads to variation in complications seen.

Mucosal membrane involvement and "target lesions" erythema multiforme are more common in Stevens-Johnson's syndrome. The most frequently affected membrane is the oropharynx followed by the eyes and genitals.

Skin biopsy may be indicated in some cases where the diagnosis is in doubt. Full thickness epidermal necrosis is seen in TEN while satellite cell necrosis may be seen in SJS. Other useful investigations include complete blood count, urinalysis, serum electrolyte and urea estimation and activated partial prothrombin time. The liver function test may also be included in some patients.

\section{Management}

Early recognition and prompt withdrawal of the causative agent and referral to a burns unit improves outcome.

- $\quad$ Aggressive fluid and electrolyte management is essential. Patients with respiratory involvement should be monitored carefully to prevent pulmonary oedema. Marked vasodilation of blood vessels may lead to high out put failure in the elderly.

Respiratory distress may result from mucosal sloughing and may necessitate endotracheal intubation.

- Meticulous skin care is mandatory. Areas of skin erosion in TEN should be covered with non-adherent protective dressing such as petroleum gauze, preferably sterile.

Silver sulphadiazine should be avoided because it is a sulphonamide derivative and may precipitate TEN.

Drug use is minimized as cross reactions to other drugs could also worsen the skin reaction. Although it is said that antibiotics are useful only when sepsis is strongly considered, it is advisable to use antibiotics routinely in patients in this environment as there is usually some bacterial infection of the skin. The use of steroids in this group of patients remains controversial. Theoretically steroids inhibit further immune complex reactions in the skin, however immune suppression from the steroids reduce the ability of the patient to fight infection. However, steroids may be beneficial to the patient if new lesions continue to develop. The use of intravenous immunoglobulin G(IVIG) in these patients remains controversial. Some reports demonstrate favourable outcome. Immediate discontinuation of offending drugs should be emphasized.

Other specialist such as the ophthalmologists, plastic surgeons, respiratory physicians and gastroenterologists should be involved in the management of patients when indicated. 


\section{Complications}

Sepsis, pain and scarring of mucosal membranes occur in many cases. Dry eyes, entropion, vulvovaginal synechiae and phimosis have been reported.

Gastrointestinal haemorrhage may result from intestinal inflammation. Acute renal insufficiency results from poor intake and septic shock which may induce renal hypoperfusion.

Prognosis in TEN is poor with mortality rates as high as $40 \%$.

\section{PEMPHIGUS VULGARIS}

Pemphigus vulgaris (PV) is an autoimmune disorder characterized by generalized intraepidermal blistering of the skin accentuated initially in intertriginous regions $[10,11]$.

\section{Pathogenesis}

Pemphigus vulgaris results from autoimmunity to the pemphigus vulgaris antigen [12]. In addition lower molecular weight species have been extracted form human and other vertebrate epithelial tissues which are also identified by circulating autoantibodies from patients with this disease [13]. Pemphigus vulgaris has been reproduced at histologic, immunologic and clinical levels when pemphigus vulgaris IgG was infused intraperitoneally into new born mice [14]. It is believed that acantholysis is the end result of plasmin induced proteolysis which results from binding of pemphigus autoantibody to pemphigus antigen on keratinocyte cell membranes, production and release of plasminogen activator and subsequent conversion of plasminogen to plasmin within the affected epidermis.

\section{Clinical Features}

Patients with PV are usually quite ill. Fever, chills, rigours, and head ache are not uncommon. Mucous membrane involvement is also common manifesting with sore throat, dysphagia, dysuria and vaginal pain. When large areas of the skin gets denuded from ruptured blisters there may be marked fluid and protein losses leading to severe electrolyte imbalance, hypoalbuminemia and development of life-threatening secondary bacterial infections.

\section{Management}

Pemphigus vulgaris may be rapidly fatal without treatment. Large doses of corticosteroids are usually required. Oral daily doses of corticosteroids may range between 120-150mg of prednisolone or $1 \mathrm{gm}$ of methyl prednisolone daily in the first 3-5 days. Most patients are treated with corticosteroids and a steroid sparing immunosuppressant e.g. azathioprine or cyclosphosphamide. Plasmapheresis has been used in conjunction with immunosuppressant therapy to prevent autoantibody rebound. This may be in addition to intravenous pulse therapy with high dose steroids [15]. Results of various therapies vary [16].

Antibiotics are also required to prevent overwhelming infections. Fluid and electrolyte balance should also be managed carefully. It is also important to remember that certain drugs could precipitated pemphigus and should be avoided in such patients.

\section{STAPHYLOCCOCAL SCALDED SKIN SYNDROME (RITTER'S DISEASE)}

This condition is commonly seen in infants and children. It is caused by Staphyloccus aureus phage type 71 due to liberation of exotoxin [17].

The clinical features include diffuse erythema, fever, tender skin, large flaccid bullae with clear fluid which rupture soon after being formed. This may lead to extensive loss of the skin surface. Lesions usually start in the head and migrate downwards [18]. In Staphylococcal scaled skin syndrome, the exfoliative toxins are spread haematogenously from a localized source causing widespread epidermal damage at distant sites [17]. Patients should be admitted preferably in a burns unit. The lesions should be managed like those other conditions listed above. Fluid and electrolyte balance must be monitored and appropriate antibiotics should be used. It is associated with high mortality

\section{LEPRA REACTIONS}

Lepra reactions occur in the course of leprosy. They are sudden exacerbations in the clinical state of a patient with pauci or multibacillary leprosy[19]. These reactions are treated as emergencies as they may lead to irreversible damage to nerves leading to loss of functions of limbs or blindness. Sometimes 
lepra reactions are due to immunological changes following effective treatment and reduction in bacillary load [19]. Other factors that may provoke leprae reactions include intercurrent infections with malaria or viruses. Lepra reactions are of two types.

\section{Type 1 reaction}

This is an example of type IV hypersensitivity reaction. It involves cell mediated immunity and is seen in the entire spectrum of leprosy except lepromatous type [19]. It manifests as a local aggravation of an existing lesion which become more erythematous and indurated with increased loss of sensation. The lesions may be painful with paraesthesis. The nerve may become extremely painful and tender. When it is due to increase in cell mediated immunity, the term reversal or upgrading is used. It occurs during treatment. When it is associated with decrease in cell mediated iummunity, the word "downgrading" is used. Downgrading reactions occur in a patient not on treatment.

\section{Type II reaction}

This reaction is also known as erythema nodosum leprosum (ENL). It is mediated by humoral hypersensitivity. Usually occurs later during the course of treatment for lepromatous and borderline lepromatous leprosy. Type II reaction manifests as recurrent, tender erythematous nodules, appearing suddenly on any part of the body. Other features like joint pain, fever, malaise may be present [19].

\section{Treatment}

Type 1 reactions should be treated with systemic corticosteroids especially when there is nerve involvement. Non steroidal anti inflammatory drugs are also useful.

Type II reactions require the use of oral steroids or clofazimine. Thalidomide in a dose of $100 \mathrm{mg}$ to $400 \mathrm{mg}$ daily has also been found to be effective.

\section{ANGIOEDEMA}

Urticaria and angioedema are common cutaneous vascular reaction patterns. Urticaria is characterised by transient, pruritic edematous, lightly erythematous papules or wheals frequently with central clearing which may vary in size from a few to many centimetres in diameter. The individual lesions are transient lasting 12 to 24 hours or less [19].

Angioedema is the term used to describe a process that is in most respect similar to urticaria but involves deeper subcutaneous structures [19]. Both conditions may occur in the same individual. Life threatening reactions are associated with angioedema especially when the respiratory mucous membranes are involved leading to laryngeal oedema. Severe attacks may be associated with abdominal pain, nausea, vomiting due to intestinal obstruction in children. Headaches and high fever may also occur in children. These are all mediated by histamine. Most cases are Type 1 hypersensitivity reactions which are mediated by IgE antibodies fixed on the surface of tissue mast cells and basophils. As a result of antigen-antibody reaction on mast cells, degranulation of mast cells occur leading to the release of histamine which causes itching, vasodilatation, and increased permeability of blood vessels resulting in the formation of wheals. Sometimes urticaria is due to Type III hypersensitivity reaction and is mediated by IgG antibodies which form immune complexes with antigens leading to release of vasoactive substances from complement components which release histamine with the formation of wheals. A Type III mechanism is implicated in urticarial vasculitis.

Factors implicated in urticaria include anaphylatoxins (C3a and C5a), kinins, serotonin, prostaglandins, fibrin degradation products, and substance $P$ [20]. Genetic factors are implicated in some rare types of urticaria and angioedema. Hereditary angioedema, familial cold urticaria, vibratory angioedema, and Muckle-Wells syndrome (a heredo-familial syndrome of urticaria, deafness and amyloidosis). The role of $\mathrm{C} 1$ esterase inhibitor deficiency in hereditary angioedema is one of the best examples. Some patients with cold intolerance have been found to have reduced alpha-1antirypsin and chemotrypsin activity[21]. Patients on angiotensin converting enzyme inhibitors may also develop angioedema. 

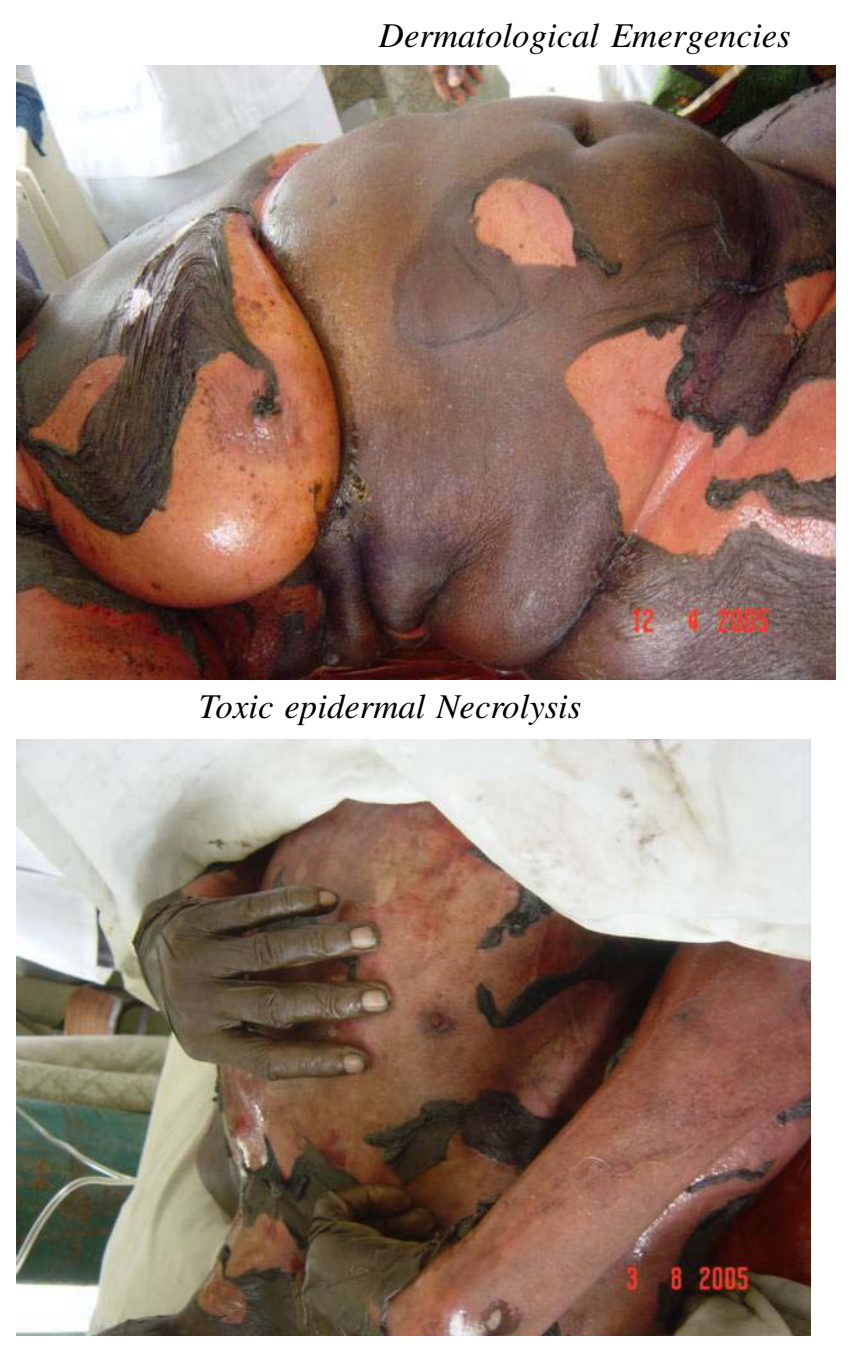

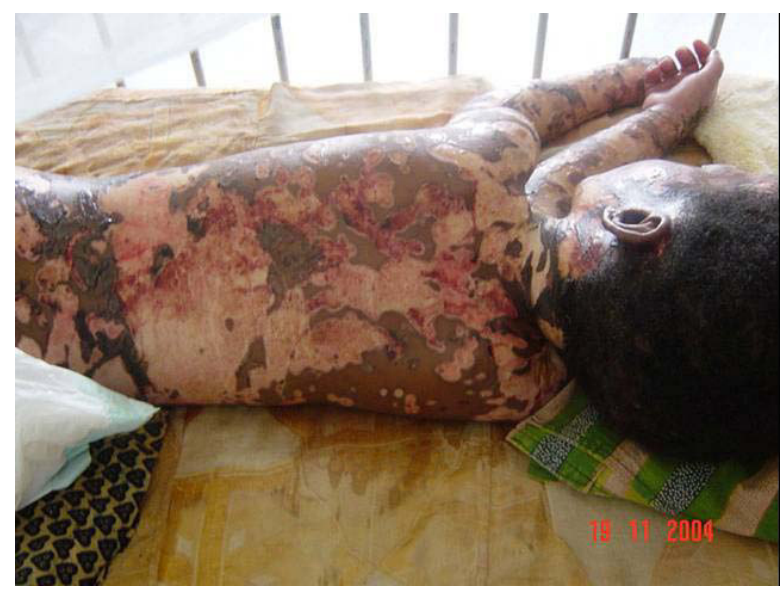

Stevens-Johnson Syndrome in a child

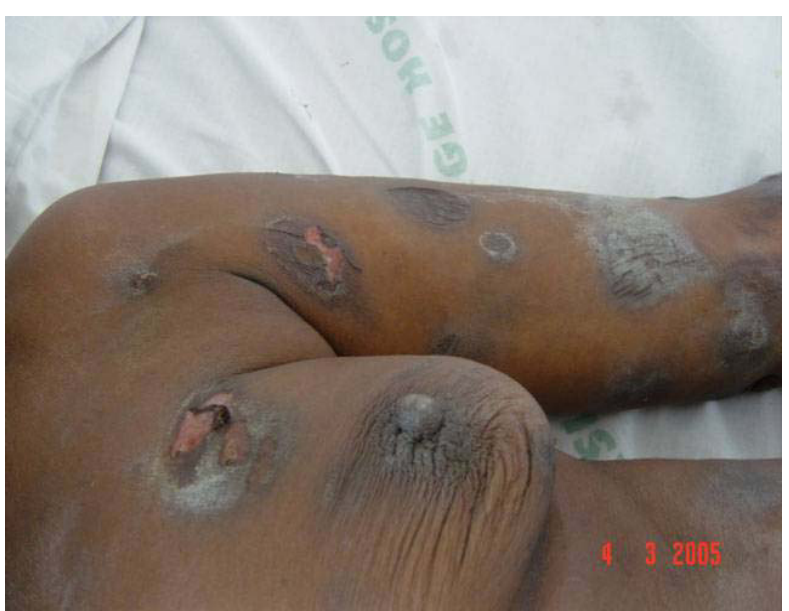

Stevens-Johnson Syndrome with target lesions 


\section{Dermatological Emergencies}

\section{Management}

Angioedema of the glaborous skin is usually not dangerous. However involvement of the mucous membrane may be dangerous. Such patients may present with complains of lumps in their throats or difficulty with breathing. Such patients should be observed carefully.

Parenteral Corticosteroids or epinephrine may be used in severe attacks. Danazol has also been found to be very useful. If due to ACE inhibitors, this must be stopped.

Traditional anti-histamines of the $\mathrm{H} 1$ type are useful in prophylactic management. Side effects should be looked out for. Newer antihistamines with non-sedating effects are preferred. Non-sedating H1 anti-histamines e.g. terfenadine, astemizole and loratadine.

Some tricyclic antidepressants have been found useful in chronic urticaria because they exert potent $\mathrm{H} 1$ antihistamine effects [22].

Detailed history is very important. Suspected urticaria due to a food item can be confirmed by disappearance of the urticaria within $48 \mathrm{hrs}$ after stoppage of the particular food (diet elimination test) or by giving a certain food with recurrence of the urticaria (provocation test).

\section{CONCLUSION}

Dermatologic emergencies are important clinical conditions, which contribute significantly to morbidity and mortality in health care delivery. Although various pathophysiologic mechanisms are involved in the occurrence of these lesions they may ultimately lead to skin failure and consequently complications of the skin. Dermatologic emergencies should therefore be earlier identified as such and should be given the urgency required in management to prevent avoidable mortality which arises from a number of these conditions where possible.

\section{REFERENCES}

1. Irvin C. Skin failure - a real entity, discussion paper. J R Soc. Med 1991; 84: 412-413.

2. Ryan JJ. Disability in Dermatology. Br J. Hosp Med 1991; 46: 33-36.

3. Lyell A. toxic epidermal necrolysis. An eruption resembling scalding of the skin. Br. J.
Dermatol 1956; 68: 355-361.

4. Lyell A. toxic epidermal necrolysis (The scalded skin syndrome) a re-appraisal. Br. J. Dermatol. 1979: 100(1); 69-86.

5. Ayangco L and Rogers RS. Oral manifestations of erythema multiforme. Dermatol Clin. 2003; 21(1): 195-197.

6. Prendville J. Stevens-Johnsons Syndrome and toxic epidermal necrolysis. Adv. Dermatol 2002; 18 : 151-73.

7. Rojeau JC, Guillaume JC, Fabre JP et al. Toxic epidermal necrolysis (Lyell Syndrome): Incidence and drug history in France 1981-1985. Arch Dermatol. 1990; Jan. 126(1): 37-42.

8. Chave T.A, Mortemer NJ, Sladden MJ et $a l$. Toxic Epidermal Necrolysis. Current evidence, practical management and future directions. Br. J. Dermatol 2005; 153(2): 241-53.

9. Criton S, Devi K, Sridevi PK and Asokan P. Toxic Epidermal Necrolysis - a retrospective study. Int. J. Dermatol 1997; 36(12) : 923

10. Ahmed AR. Pemphigus Vulgaris: Clinical features. Dermatol Clin. 1983; 1: 171-177.

11. Jordan RE. Pemphigus. In Fitz Patrick TB, Eisen AZ, Wolff K, et al (eds). Dermatology in General Medicine. 3rd Ed. New York McGrawHill; 1987: 571-579.

12. Stanley JR, Yaar M, Hawley-Nelson P et al. Pemphigus antibodies identify a cell surface glycoprotein synthesised by human and mouse keratocytes. J. Clin. Invest. 1982. 70: 281-288.

13. Peterson $\mathrm{Ll}$ and Winepper KD. Isolation and purification of the Porphigus Vulgaris antigen form human epidermis. J. Clin. Invest. 1984 73: 11131120.

14. Anhalt GJ, Labib RS, Voorhees JJ, et al. Induction of pemphigus in neonatal mice by passive transfer of IgG from patients with the disease. $\mathrm{N}$ Engl J.Med. 1982; 306: 1189-1196.

15. Fine JD, Appel M, Green LK et al. Pemphigus Vulgaris. Combined treatment with intravenous corticosteroid pulse therapy, plasmapheresis and azathioprine. Arch. Dermatol 1988; 124: 236-239.

16. Guillaume JC, Roujeau JC, Morel $\mathrm{P}$ et al. Controlled study of plasma exchange in pemphigus. Arch Dermatol 1988; 124: 1659-1663. 
17. Johnston GA. Treatment of bullous impetigo and the Staphylococcal Scalded Skin Syndrome. Expert review of anti-infective therapy 2004; 2(3): 439-446.

18. Patel G, Varma $S$ and Finlay AY. Staphylococcal Scalded Skin Syndrome in healthy adult. Br. J. Dermatol 2000; 142(6); 1253.

19. Gupta R. Urticaria and Angioderma. In Textbook of Dermatololgy. Jaypees. 2002: 127.
20. Czarnetzki BM. Mechanism and Mediators in Urticaria. Semin Dermatol 1987; 6: 272-285.

21. Doeglas HMA and Bleumink E. Protease inhibitors in plasma of patients with chronic urticaria. Arch Dermatol 1975, 111: 979-985.

22. Green SL, Reed CE and Schroeter AL. Double-blind crossover study comparing doxepin with diphenhydramine for the treatment of chronic urticaria. J. Am Acad Dermatol. 1985, 12: 669675. 